\title{
Remoção parcial de tecido cariado em dentes permanentes: seis anos de acompanhamento
}

\author{
Partial caries removal in permanent teeth: six-year follow-up \\ Juliana Jobim Jardim* \\ Miriam Nunes Doege Simoneti* \\ Marisa Maltz
}

\section{Resumo}

Objetivos: o objetivo deste estudo foi avaliar a efetividade da remoção parcial de tecido cariado (RPTC) de lesões de cárie profundas e restauração em uma única sessão de forma comparativa ao tratamento expectante (TE) em seis anos de acompanhamento. Materiais e método: o estudo foi realizado em Porto Alegre e Brasília. A amostra incluiu pacientes com molares permanentes com lesões de cárie profundas (metade interna de dentina), sem lesão periapical ao exame radiográfico, resposta positiva ao teste de frio, negativa à percussão e ausência de dor espontânea. Esses foram randomicamente atribuídos ao grupo teste (RPTC), recebendo remoção incompleta de tecido cariado e restauração em uma sessão, ou controle (TE). Após, os dentes foram restaurados com resina composta ou amálgama. O desfecho avaliado foi vitalidade pulpar (sensibilidade ao teste térmico, sensibilidade à percussão e ausência de alteração periapical). Análises de sobrevivência foram realizadas para estimar as taxas de sucesso do tratamento e definir variáveis associadas, utilizando o modelo de regressão de Weibull. Foram executados 299 tratamentos, sendo 152 RPTC e 147 TE. Resultados: somente o acompanhamento dos tratamentos realizados em Porto Alegre foi realizado. Após 6 anos, 127 dentes foram avaliados. Foram observadas taxas de sucesso de $60 \%$ para RPTC e $32 \%$ para TE $(p<0,05)$. Dentre as variáveis analisadas, somente a tipo de tratamento apresentou associação significativa à manutenção da vitalidade pulpar, sendo favorável à RPTC. Conclusão: RPTC pode ser alternativa viável no tratamento de lesões profundas de cárie, mostrando melhora significativa em relação à manutenção da vitalidade pulpar quando comparado ao TE.

Palavras-chave: Cárie dentária. Dentes permanentes. Remoção parcial de tecido cariado.

\section{Introdução}

O procedimento tradicional para o tratamento de lesões de cárie profundas consiste na remoção total da dentina cariada ${ }^{1}$. A remoção total do tecido cariado pode resultar em exposição pulpar. Nessas situações, o capeamento pulpar direto pode ser considerado um meio de preservar a vitalidade da polpa e estimular a formação de uma barreira calcificada para bloquear a exposição ${ }^{2}$. No entanto, as taxas de sucesso do tratamento de lesões profundas de cárie reduzem drasticamente quando ocorre exposição pulpar ${ }^{3}$. Mesmo quando realizado capeamento pulpar direto, na presença de tecido cariado, as taxas de sucesso são muito baixas: $31,8 \%$ após um ano ${ }^{3}, 37 \%$ em 5 anos de acompanhamento e $13 \%$ em 10 anos $^{4}$.

Com a finalidade de evitar a exposição pulpar, tem sido sugerida a remoção de dentina cariada em duas etapas, também chamada de tratamento expectante ou capeamento pulpar indireto ${ }^{5}$. A primeira etapa nessa técnica consiste na remoção superficial da dentina necrótica e desmineralizada e no forramento com hidróxido de cálcio, seguido de restauração provisória. Em uma segunda etapa do tratamento, é feita a remoção total de tecido cariado remanescente e, após um intervalo de 4 a 6 meses, a restauração definitiva ${ }^{6}$. O objetivo do tratamento expectante é promover uma modificação no meio ambiente da lesão, proporcionando reações fisiológicas do complexo dentino-pulpar que irão provocar uma alteração na velocidade do desenvolvimento da doença de rápida para lenta, ou mesmo paralisação

\footnotetext{
Doutora em Cardiologia/Dentística, Ufrgs, Departamento de Odontologia Preventiva e Social, Porto Alegre, RS, Brasil.
}

Cirurgiã- Dentista, Ufrgs, Departamento de Odontologia Preventiva e Social, Porto Alegre, RS, Brasil. 
de sua progressão, favorecendo a manutenção da vitalidade pulpar 7 . Essa técnica tem demonstrado resultados terapêuticos favoráveis ${ }^{7-8}$, sendo o risco de exposição pulpar reduzido comparado à remoção total de tecido cariado ${ }^{2-3,9-10}$, apresentando taxas de sucesso que variaram de $74,1 \%$ a $93,4 \%$ em 1 ano de acompanhamento ${ }^{3,11-12}$.

Apesar dos resultados favoráveis apresentados pelo TE, como a diminuição do risco de exposição pulpar e a manutenção da vitalidade pulpar, em acompanhamentos longitudinais ${ }^{9}$ encontram-se algumas desvantagens nesse tipo de tratamento. Por serem necessárias duas sessões para sua realização, o tratamento torna-se mais oneroso e causa maior desconforto ao paciente devido à necessidade de uma nova consulta. Pode também ocorrer contaminação pelo meio externo, resultante da perda da restauração provisória, ou, ainda, perda de tecido dentário íntegro pela remoção do material restaurador temporário ${ }^{13}$.

A remoção superficial de dentina cariada associada a um bom selamento da superfície faz com que ocorra redução significativa no número de micro-organismos presentes e paralisação da progressão da lesã $0^{6,14-16}$. O selamento que a restauração proporciona impede a entrada de nutrientes às bactérias remanescentes no tecido cariado, reduzindo significativamente o número de bactérias presentes na lesã $0^{17}$. Diante dessas evidências, surge o questionamento da necessidade de reabertura da cavidade para uma nova remoção de dentina cariada ${ }^{18}$.

Estudos passam a sugerir então remoção parcial de dentina cariada e restauração em uma única sessão como tratamento definitivo. Há estudos avaliando remoção parcial como tratamento definitivo em dentes permanentes, a curto prazo $^{19}$ e a longo prazo em lesões $\operatorname{rasas}^{20}$ e profundas ${ }^{21-22}$. Após dez anos de acompanhamento clínico ${ }^{22}$ e radiográfico ${ }^{21}$, verificou-se que é possível paralisar a lesão cariosa após remoção parcial de tecido cariado e restauração definitiva em única sessão e, com isso, preserva-se a vitalidade pulpar a longo prazo. Também são encontrados estudos que avaliaram remoção parcial como tratamento definitivo somente em dentição decídua, que têm menor tempo de permanência em boca, levando a um menor tempo de avaliação $0^{23-26}$.

Há necessidade de um maior número de ensaios clínicos, randomizados e controlados avaliando remoção parcial de tecido cariado como tratamento definitivo em dentes permanentes a fim de auxiliar os dentistas na tomada de decisão $0^{11,17}$. São necessários, também, estudos de acompanhamento longitudinal, pois investigações mostram que alterações pulpares podem levar tempo a serem desenvolvidas ${ }^{4,22}$.

Diante dessas observações, iniciou-se em 2005 o trabalho "Tratamento alternativo de lesões de cárie profundas - um estudo multicêntrico", com o objetivo de estudar a efetividade do tratamento alternativo de lesões profundas de cárie em Serviços de Saúde em diferentes regiões brasileiras - Porto Ale- gre (Região Sul) e Brasília (Região Centro-Oeste). A técnica alternativa consiste na restauração definitiva do dente após remoção parcial de dentina cariada em uma única sessão. Foram tratados 299 dentes nesse ensaio clínico, randomicamente alocados nos grupos controle (tratamento expectante - TE) ou teste (remoção parcial de tecido cariado - RPTC). Posteriormente, foram realizados acompanhamentos de dezoito meses, com taxas de sucesso de $98 \%$ para o grupo de tratamento alternativo proposto ${ }^{27}$, dois $^{28}$, três ${ }^{29}$ e cinco anos $^{30}$, este último apresentando taxas de sucesso de $79 \%$ para o grupo de tratamento alternativo de lesões cariosas.

O objetivo do presente estudo foi comparar a efetividade do tratamento alternativo de lesões profundas de cárie (remoção parcial de tecido cariado e restauração em uma única sessão) ao tratamento expectante, após 6 anos de acompanhamento.

\section{Materiais e método}

\section{Delineamento do estudo}

Este estudo é uma avaliação de 6 anos de um ensaio clínico, randomizado, controlado, multicêntrico (número de registro em www.clinicaltrials.gov NCT00887952). De 2005 a 2007, 299 tratamentos clínicos foram realizados por 22 cirurgiões-dentistas treinados especificamente para o estudo e supervisionados por cinco pesquisadores principais. Esses tratamentos foram realizados em dois centros localizados nas cidades de Porto Alegre e Brasília, em unidades de serviço de saúde pública ou de universidades federais.

\section{Amostra}

O tamanho da amostra foi calculado com base na diferença entre as taxas de sucesso para cada tratamento, após um período de 5 anos de avaliação (TE - 60,9\% e RPTC - 82\%), em $\alpha=5 \%$, com um poder de $90 \%{ }^{16,31}$. Isso resultou na necessidade de 76 restaurações por grupo de tratamento. O número de restaurações foi aumentado para 119 por grupo quando se considerou uma taxa de abandono de $56 \%$ após 2 anos $^{32}$.

Os participantes foram selecionados entre os frequentadores regulares dos serviços de saúde pública ou por meio de uma busca ativa de pessoas que poderiam cumprir os critérios de inclusão, em programas comunitários, escolas locais e pela propaganda em jornal e rádio. A amostra foi composta por pacientes com lesões profundas de cárie em molares permanentes. Os critérios de seleção incluíram: molares com lesões profundas de cárie (atingindo metade interna de dentina ou mais ao exame radiográfico); resposta positiva ao teste de frio $\left(-20^{\circ}\right.$ C gás refrigerado - Aerojet, Rio de Janeiro, RJ, Brasil); resposta negativa à percussão; ausência de dor 
espontânea e ausência de lesão periapical (exame radiográfico). Os pacientes foram excluídos ao apresentarem perda de cúspide ou cárie abaixo da margem gengival.

Todos os indivíduos foram informados dos riscos e propósitos da pesquisa e assinaram um termo de consentimento. Os participantes receberam atendimento odontológico pelos pesquisadores ao longo de sua participação neste estudo. Eles não receberam incentivos financeiros visto que os serviços de saúde pública no Brasil são gratuitos. O estudo foi aprovado pelo Comitê de Ética em Pesquisa da Universidade Federal do Rio Grande do Sul (protocolo 18/05), pelo Comitê de Ética Municipal de Porto Alegre (protocolo 27/06), pelo Comitê de Ética do Grupo Hospitalar Conceição (Protocolo 070/05) e pelo Comitê de Ética do Hospital Universitário de Brasília (protocolo 045/2005).

\section{Grupos de estudo}

Os pacientes foram distribuídos aleatoriamente para os grupos teste ou controle. O grupo controle recebeu o tratamento expectante, enquanto o grupo teste recebeu remoção parcial da cárie seguida por restauração em uma única sessão. Ambos os grupos foram novamente divididos de acordo com o material restaurador: amálgama ou resina composta.

\section{Randomização e cegamento}

A unidade de randomização foi o dente e a sua inclusão para os grupos teste ou controle foi feito da seguinte forma: o grupo de tratamento foi escrito em um pedaço de papel numerado e mantido em um frasco escuro, uma pessoa que não fosse o dentista deveria selecionar um pedaço de papel a partir do frasco e o tratamento era então realizado como indicado. $\mathrm{O}$ material restaurador foi determinado pela alternância entre amálgama e resina composta a cada duas semanas. O cegamento dos pacientes não foi possível devido ao diferente número de consultas necessárias para cada tratamento. O operador estava cego durante todo o processo até a randomização, a fim de evitar possível influência no processo de remoção da dentina cariada em ambos os grupos. A avaliação do resultado do tratamento no acompanhamento de 6 anos também foi feita seguindo o princípio de cegamento. Os dados foram registrados em fichas clínicas e, em seguida, transferidos para um sistema digital on-line (http://odonto.cityzoom.net).

\section{Procedimentos clínicos}

Todos os tratamentos realizados seguiram o mesmo protocolo, como segue: anestesia local e isolamento absoluto; acesso à lesão utilizando pontas diamantadas, se necessário; remoção completa do tecido cariado das paredes circundantes da cavidade com colher de dentina e/ou brocas de baixa ro- tação, de acordo com critério clínico de dureza (resistência à sondagem); remoção parcial cuidadosa da cárie da parede pulpar (apenas tecido mole desorganizado foi removido); limpeza da cavidade com água destilada e secagem com filtro de papel estéril; randomização dos grupos. Dentes atribuídos ao grupo controle (TE) receberam capeamento pulpar indireto com cimento de hidróxido de cálcio (Dycal, calafetar / Dentsply, Rio de Janeiro, RJ, Brasil) e restauração temporária com cimento de óxido de zinco e eugenol modificado (IRM, calafetar / Dentsply, Rio de Janeiro, RJ, Brasil ), a cavidade foi então reaberta, após um tempo médio de 90 dias, (percentil $25=60$ dias; percentil $75=150$ dias, média 120 \pm 120 dias), o tecido mole cariado remanescente foi removido e o dente restaurado com cimento de ionômero de vidro (Vitro Fil, DFL, Rio de Janeiro, RJ, Brasil), e amálgama (SDI, Bayswater WA, Austrália) ou resina composta (TetricEvoCeram, Ivoclar / Vivadent, Liechtenstein). Dentes alocados ao grupo teste (RPTC) receberam imediatamente restauração com cimento de ionômero de vidro e amálgama ou resina composta após remoção de tecido cariado.

\section{Resultados de acompanhamento}

O sucesso do tratamento foi avaliado por meio da manutenção da vitalidade pulpar, considerada um desfecho combinado em dentes com resposta positiva ao teste térmico, resposta negativa à percussão, ausência de dor espontânea e ausência de lesão periapical (exame radiográfico). Pacientes com perda de vitalidade pulpar anterior ao acompanhamento não foram avaliados.

\section{Análise estatística}

Modelos paramétricos de sobrevivência com nível de fragilidade individual foram utilizados para o resultado com o tipo de tratamento como fator exploratório. Um ajuste foi feito para preditores potenciais, tais como idade, sexo, região, material restaurador e número de superfícies restauradas. Análises de sobrevivência foram realizadas utilizando o teste "goodness-of-fit" com probabilidade estatística para estimar as taxas de sucesso do tratamento. O modelo de regressão de Weibull foi utilizado para comparar as curvas de sobrevivência, considerando a dependência das observações quando mais de um tratamento foi realizado em um único indivíduo. Observação censurada (dados faltantes) foi estipulada para todos os pacientes perdidos no acompanhamento. Todos os pacientes avaliados contribuíram ao menos uma vez para a taxa de sobrevivência. $\mathrm{O}$ tempo para o resultado avaliado foi contado e analisado em dias. O nível de significância foi fixado em 5\% e a unidade de análise foi o dente. Todas as análises foram realizadas com o software STATA, versão 12.0. 


\section{Resulltados}

Neste estudo, somente o acompanhamento dos tratamentos realizados em Porto Alegre foi realizado e analisado. Durante 6 anos de acompanhamento, 127 dentes foram avaliados. Dos 64 dentes avaliados do grupo RPTC, houve 18 insucessos e 46 sucessos. Do grupo TE foram avaliados 63 dentes, dos quais 29 apresentaram insucesso e 34 sucesso. Foram observadas taxas de sucesso de $60 \%$ para o grupo RPTC e $32 \%$ para o grupo TE $(\mathrm{p}<0,05)$ (Figura 1).

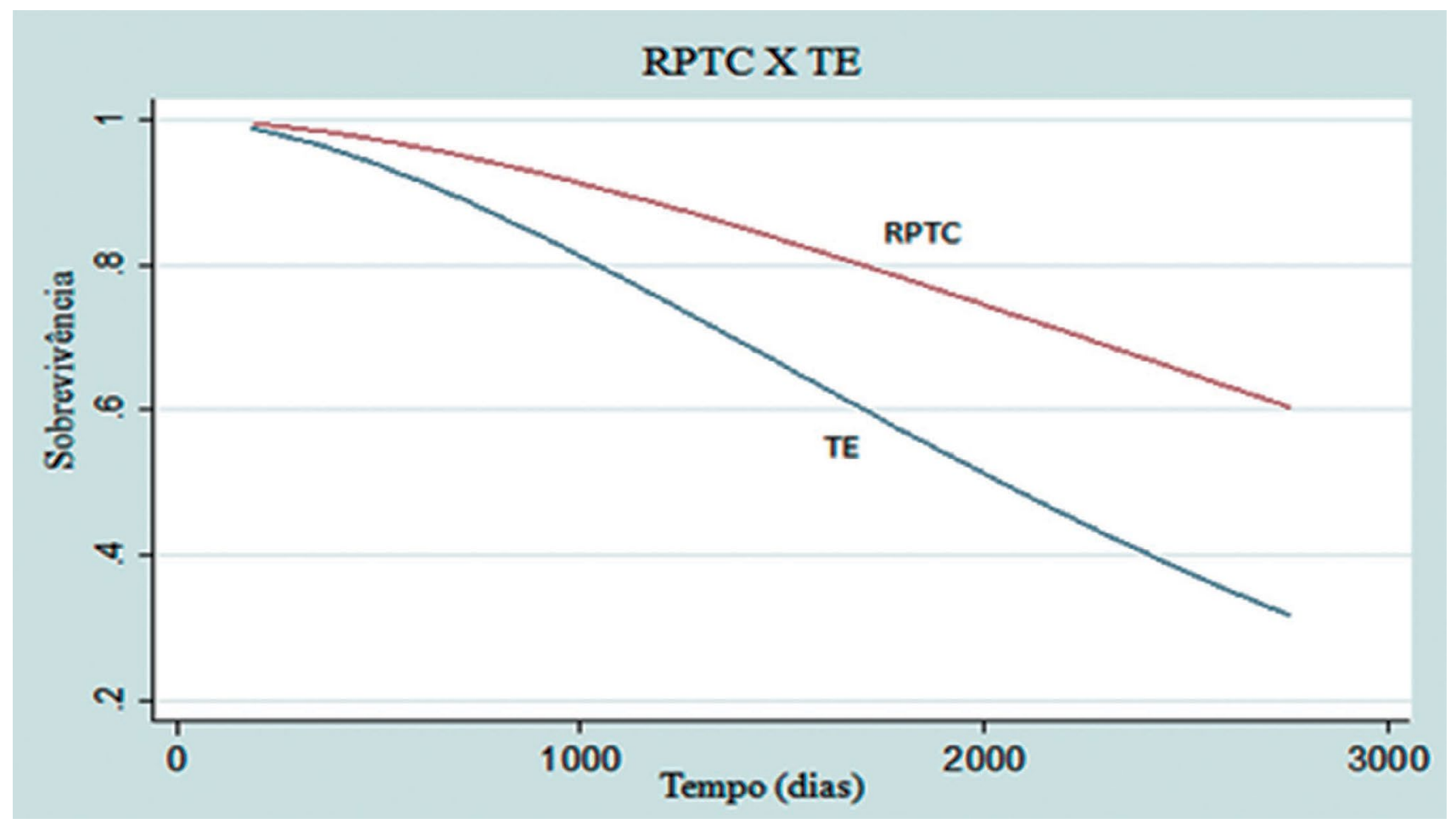

Figura 1 - Taxas de sucesso para os tratamentos RPTC X TE (modelo de regressão de Weibull)

Dentre as variáveis analisadas, somente tipo de tratamento apresentou associação significativa com o desfecho de manutenção da vitalidade pulpar. $\mathrm{O}$ desfecho foi favorável quando o tratamento RPTC foi comparado ao TE, apresentando-se como um fator de proteção (OR 0,44 p=0.007), ou seja, quem recebe esse tratamento tem $56 \%$ menos chance de insucesso pulpar do que quem recebe o tratamento expectante. Idade, gênero e tipo de material restaurador não apresentaram associação com o desfecho (Tabela 1).

Tabela 1 - Associação entre necrose pulpar e variáveis analisadas (regressão de Weibull)

\begin{tabular}{|c|c|c|c|c|}
\hline \multirow{2}{*}{ Variáveis } & \multirow[b]{2}{*}{$\mathrm{N}(\%)$} & \multicolumn{3}{|c|}{ Regressão Multivariada de Weibull } \\
\hline & & $R R$ & $95 \%$ IC & $\mathrm{p}$ \\
\hline \multicolumn{5}{|l|}{ Gênero } \\
\hline Masculino & $47(37 \%)$ & 1.00 & & \\
\hline Feminino & $80(63 \%)$ & 1.11 & $0.54-2.28$ & 0.77 \\
\hline \multicolumn{5}{|l|}{ Idade } \\
\hline$\leq 17$ anos & $73(57.5 \%)$ & 1.00 & & \\
\hline$>17$ anos & $54(42.5 \%)$ & 0.67 & $0.35-1.29$ & 0.23 \\
\hline \multicolumn{5}{|l|}{ Tratamento } \\
\hline Tratamento expectante & $63(50 \%)$ & 1.00 & & \\
\hline Remoção parcial & $64(50 \%)$ & 0.44 & $0.24-0.80$ & 0.007 \\
\hline \multicolumn{5}{|l|}{ Material restaurador } \\
\hline Amálgama & $30(24 \%)$ & 1.00 & & \\
\hline Resina Composta & $97(76 \%)$ & 0.67 & $0.36-1.26$ & 0.21 \\
\hline
\end{tabular}


Dos 63 dentes avaliados do grupo TE, 44 receberam tratamento expectante completo e dezenove ficaram incompletos, sendo os incompletos aqueles tratamentos em que o paciente não retornou em 90 dias para a conclusão do tratamento (remoção do tecido cariado remanescente e restauração definitiva). Dos dezenove incompletos, dezesseis apresentaram insucesso após 6 anos de acompanhamento, sendo a taxa de sucesso para o expectante incompleto de $3 \%$ apenas, enquanto que os que receberam expectante completo apresentaram uma taxa de sucesso de $57 \%$ (Figura 2). Foi observado que o tratamento expectante incompleto tem uma chance de insucesso 6,4 vezes maior do que o completo (ajustado para as outras variáveis).

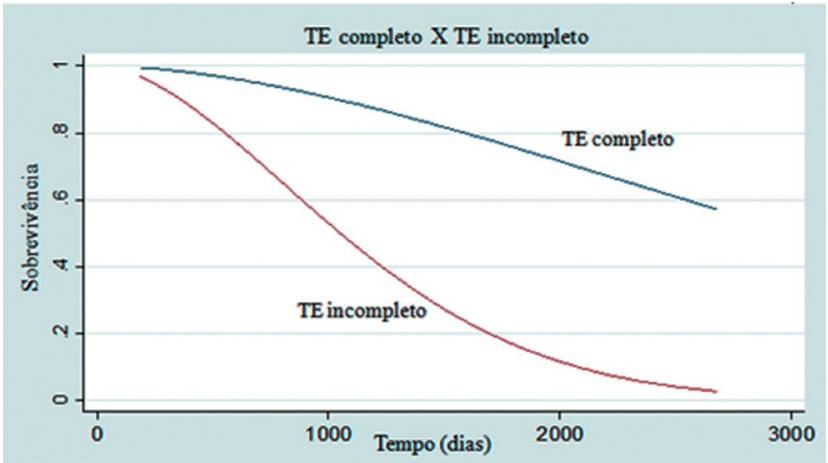

Figura 2 - Taxas de sucesso para os tratamentos TE completos $X$ incompletos (modelo de regressão de Weibull).

\section{Discussão}

Este estudo comparou o desempenho dos tratamentos por meio de RPTC e TE realizados em lesões profundas de cárie quanto à manutenção da vitalidade pulpar em 6 anos de acompanhamento. Houve uma diferença significativa entre as taxas de sucesso dos tratamentos propostos $(\mathrm{p}<0,05)$. Após 6 anos de acompanhamento, os resultados mostram que RPTC é mais efetivo em preservar a vitalidade pulpar do que TE.

Muitos estudos avaliaram remoção parcial em dentes decíduos como tratamento definitivo e encontraram resultados favoráveis à $\mathrm{RPTC}^{23-25}$. Estudos em dentes permanentes também encontraram resultados favoráveis para RPTC, avaliando lesões ra$\operatorname{sas}^{20}$ e lesões profundas ${ }^{21-22}$, sendo as taxas de sucesso deste de $97 \%, 90 \%, 82 \%$ e $63 \%$ para RPTC após um ano e meio, três, cinco e dez anos de acompanhamento. Resultados semelhantes foram encontrados no presente estudo randomizado: $99 \%, 91 \%, 78 \%$ e $60 \%$ de sucesso em um ano e meio, três, cinco e seis anos de acompanhamento, sendo o último dado só para a região de Porto Alegre. Além disso, o desfecho foi favorável quando o tratamento RPTC foi comparado ao TE, apresentando-se como um fator de proteção (OR 0,44 p=0.007), ou seja, quem recebe esse tratamento tem $56 \%$ menos chance de insucesso pulpar do que quem recebe o tratamento expectante.
O tratamento expectante tem sido amplamente utilizado durante muitos anos no tratamento de lesões de cárie profundas a fim de evitar a exposição da polpa ${ }^{5,9}$. Este tratamento apresenta resultados favoráveis quando comparado aos resultados do capeamento pulpar direto, uma alternativa para quando há exposição pulpar ${ }^{3}$, a qual é uma escolha muito menos eficaz para o tratamento de lesões profundas de cárie apresentando baixas taxas de sucesso, $44,5 \%$ e $13 \%$ após 5 e 10 anos de acompanhamento respectivamente ${ }^{4}$. Em um ano de acompanhamento, o sucesso do tratamento expectante variou entre $74.1 \%^{3}$ e $93,4 \%{ }^{7}$, resultados semelhantes ao encontrado no presente estudo (86\% de sucesso após 18 meses). Após 5 anos de acompanhamento, encontramos resultados mais favoráveis para TE (53\%), do que aqueles encontrados depois do capeamento pulpar direto em dentes com lesões de cárie profundas $(31,8 \%)$, ressaltando que esse resultado foi obtido após apenas 1 ano de acompanhamento ${ }^{3}$.

Nosso estudo revelou uma diferença estatisticamente significativa $(\mathrm{p}<0.05)$ nos resultados do TE quando completos e quando incompletos $(57 \%$ e $3 \%$ de sucesso respectivamente). Mesmo sendo o risco de exposição pulpar diminuído quando realizado $\mathrm{TE}^{2}$ comparado à remoção total de tecido cariado, as chances de insucesso podem ainda assim ser altas, devido a outro fator que envolve esse tipo de tratamento: a necessidade de duas consultas para sua conclusão. O não retorno do paciente que pode resultar em falha da restauração provisória ${ }^{33}$ ou na impossibilidade de completar-se o tratamento, reduzindo-se assim as chances de sucesso desse ${ }^{29}$. No presente estudo, o não comparecimento do paciente para a segunda consulta também foi predominante, sendo que quarenta e seis pacientes não retornaram para concluir o tratamento.

A análise de regressão indicou associação entre o sucesso do tratamento e o tipo de tratamento apenas. A idade não interferiu no desfecho pulpar, assim, podemos indicar o tratamento para qualquer idade, não só para pacientes jovens. Bjorndal e Thylstrup $^{7}$ (1998) não encontraram nenhuma relação entre a idade e o sucesso do tratamento, depois de 1 ano de acompanhamento, ao realizarem TE em pacientes na faixa etária de 11-65 anos. O tipo de material restaurador também não interferiu nos resultados, podendo ser executado tanto com amálgama quanto com resina composta, sendo possível a realização da técnica em locais em que resina composta não é uma alternativa.

Os resultados mostram que quem recebe RPTC tem 56\% menos chance de insucesso pulpar do que quem recebe o tratamento expectante. Isso deve ser considerado nas tomadas de decisões porque pesquisadores e clínicos ainda estão relutantes em indicar remoção incompleta de tecido cariado na prática diária. Em um estudo realizado em Serviços Públicos de Saúde em Porto Alegre (Brasil), foram avaliadas as decisões de tratamento para lesões pro- 
fundas de cárie. A maioria dos profissionais optou por remoção completa para tratamento de lesões de cárie profunda $(71,1 \%)$, o que apresenta maior risco de exposição pulpar e, consequentemente, o pior prognóstico ${ }^{34}$. O tratamento restaurador de lesões profundas de cárie pode resultar em exposição pulpar em presença de tecido cariado resultando na necessidade de tratamento endodôntico ${ }^{13}$. O Levantamento Nacional de Saúde Bucal - SB Brasil de 2003 mostrou que aproximadamente $20 \%$ da população brasileira já havia perdido todos os dentes ${ }^{35}$. Devido à restrita oferta do tratamento endodôntico no serviço público e seu alto custo, essa situação é precursora da perda dentária de um expressivo contingente populacional ${ }^{13}$. Diante disso, o tratamento por RPTC seria uma alternativa interessante na preservação de mais dentes em boca, e por esta ser uma técnica simples, poderia ser realizada na atenção primária, resolvendo-se o problema nesse nível de atenção.

\section{Conclusão}

Os resultados sugerem que a RPTC pode ser alternativa viável no tratamento de lesões profundas de cárie, mostrando melhora significativa em relação à manutenção da vitalidade pulpar quando comparado ao tratamento expectante.

\section{Agradecimentos}

Agradecemos o apoio da Capes, $\mathrm{CNPq}(40,3420$ / 04-0), Fapergs (04 / 1531-8), e das indústrias Ivoclar / Vivadent (Schaan, Liechtenstein), DFL (Rio de Janeiro, Brasil), SDI (Bayswater WA, Austrália), e Hu - Friedy (Chicago, IL, EUA).

\section{Abstract}

Objectives: the aim of this study was to assess the effectiveness of partial caries removal (PCR) of deep caries lesions and restoration in a single session compared to stepwise excavation (SW), after six years of follow-up. Materials and method: the study was conducted in the cities of Porto Alegre and Brasilia, Brazil. The sample included patients with permanent molars with deep caries lesions (inner half of dentin), without periapical lesion upon radiographic exam, positive response to cold test, negative to percussion and absence of spontaneous pain. These were randomly assigned to the test group $(P C R)$, with incomplete caries removal and restoration in a single session, or control (SW). Then, teeth were restored with composite resin or amalgam. The outcome assessed was pulp vitality (sensitivity to cold test, sensitivity to percussion, and absence of periapical lesion). Survival analyzes were performed to estimate the rates of treatment success and define associated variables using the Weibull regression model. Two hundred ninety-nine (299) treatments were performed, with 152 PCR and 147 SW. Results: follow-up was performed only for treatments in Porto Alegre. After 6 years, 127 teeth were assessed. Success rates of $60 \%$ for PCR and $32 \%$ for SW $(p<0.05)$ were observed. Among the variables analyzed, only type of treatment was significantly associated with the maintenance of pulp vitality, being favorable to PCR. Conclusion: PCR may be a viable alternative in the treatment of deep carious lesions, showing significant improvement regarding the maintenance of pulp vitality when compared to SW.

Keywords: Dental caries. Permanent teeth. Partial caries removal.

\section{Referências}

1. Canby CP, Burnett GW. Clinical management of deep carious lesions. Oral Surg Oral Med Oral Pathol 1963;16(8):9991011.

2. Ricketts, D. Management of the deep carious lesion and the vital pulp dentine complex. British Dent J 2001;191(11):606610.

3. Bjørndal L, Reit C, Bruun G, Markvart M, Kjaeldgaard M, Näsman P, et al. Treatment of deep caries lesions in adults: Randomized clinical trials comparing stepwise vs. Direct complete excavation, and direct pulp capping vs. Partial pulpotomy. Eur J Oral Sci 2010;118:290-7.

4. Barthel CR, Rosenkranz B, Leuenberg A, Roulet JF. Pulp capping of carious exposures: treatment outcome after 5 and 10 years: a retrospective study. J Endod 2000;26(9):525-8.

5. Magnusson BO, Sundell SO. Stepwise excavation of deep carious lesions in primary molars. J Int Assoc Dent Child 1977;8:36-40.

6. Bjørndal L, Larsen T. Changes in the cultivable flora in deep carious lesions following a stepwise excavation procedure. Caries Res 2000;34(6):502-8.

7. Bjørndal L, Thylstrup A. A practice-based study on stepwise excavation of deep carious lesions in permanent teeth: a 1-year follow-up study. Com Dent Oral Epidemiol 1998;26(2):122-8.

8. Bjørndal L, Larsen T, Thylstrup A. A clinical and microbiological study of deep carious lesions during stepwise excavation using long treatment intervals. Caries Res 1997;31:411-7.

9. Leksell E, Ridell K, Cvek M, Mejàre I. Pulp exposure after stepwise versus direct complete excavation of deep carious lesions in young posterior permanent teeth. Endod Dent Traumatol 1996;12(4):192-6.

10. Schwendicke F, Stolpe M, Meyer-Lueckel H, Paris S, Dorfer CE. Cost-effectiveness of one- and two-step incomplete and complete excavations. J Dent Res 2013;92(10):880-87.

11. Bjørndal L. Indirect pulp therapy and stepwise excavation. J Endod 2008;34(7 Suppl):S29-33.

12. Orhan AI, Oz FT, Ozcelik B, Orhan K. A clinical and microbiological comparative study of deep carious lesion treatment in deciduous and young permanent molars. Clin Oral Invest 2008;12(4):369-78.

13. Jardim JJ. Partial caries removal in permanent teeth $[\mathrm{PhD}$ Thesis]. Porto Alegre, Rio Grande do Sul, Brasil: Universidade Federal do Rio Grande do Sul; 2010 [acesso em: 22 mar. 2014]. Disponível em: <http://www.lume.ufrgs.br/bitstream/handle/10183/24665/000747912.pdf?sequence $=1>$.

14. King JB, Crawford JJ, Lindahl RL, Hill C. Indirect pulp capping: A bacteriologic study of deep carious dentine in human teeth. Oral Surg Oral Med Oral Pathol 1965;20:663-71. 
15. Maltz M, Oliveira EF de, Fontanella V, Bianchi R. A clinical, microbiologic, and radiographic study of deep caries lesions after incomplete caries removal. Quintessence Int 2002;33:151-9.

16. Maltz M, Oliveira EF, Fontanella V, Carminatti G. Deep caries lesions after incomplete dentine caries removal: 40-month follow-up study. Caries Res 2007;41:493-6.

17. Ricketts DN, Kidd EA, Innes N, Clarkson J. Complete or ultraconservative removal of decayed tissue in unfilled teeth. Cochrane Database Syst Rev, Aust Dent J 2009;54(3):274376.

18. Kidd EA. How 'clean' must a cavity be before restoration? Caries Res 2004;38(3):305-13.

19. Orhan AI, Oz FT, Orhan K. Pulp exposure occurrence and outcomes after 1- or 2-visit indirect pulp therapy vs complete caries removal in primary and permanent molars. Pediatr Dent 2010;32(4):347-55.

20. Mertz-Fairhurst E, Curtis JJ, Ergle JW, Rueggeberg FA, Adair SM. Ultraconservative and cariostatic sealed restorations: results at year 10. J Am Dent Assoc 1998;129:55-66.

21. Alves LS, Fontanella V, Damo AC, Ferreira de Oliveira E, Maltz M. Qualitative and quantitative radiographic assessment of sealed carious dentin: A 10-year prospective study. Oral Surg Oral Med Oral Pathol Oral Radiol Endod 2010;109:135-41.

22. Maltz M, Alves LS, Jardim JJ, Moura MS, Oliveira EF. Incomplete caries removal in deep lesions: a 10-year prospective study. Am J Dent 2011;24(4):211-4.

23. Falster CA, Araújo FB, Straffon LH, Nör JE. Indirect pulp treatment: in vivo outcomes of an adhesive resin system vs calcium hydroxide for protection of the dentin-pulp complex. Pediatr Dent 2002;24(3):241-8.

24. Marchi JJ, de Araújo FB, Fröner AM, Straffon LH, Nör JE. Indirect pulp capping in the primary dentition: A 4 year follow-up study. J Clin Pediatr Dent 2006;31:68-71.

25. Marchi JJ, Froner AM, Alves HL, Bergmann CP, Araújo FB. Analysis of primary tooth dentin after indirect pulp capping. J Dent Child 2008;75:295-300.

26. Souza-Pinto A, Araújo FB, Franzon R, Figueiredo MC, Henz S, Garcia-Godoy F, et al. Clinical and microbiological effect of calcium hydroxide protection in indirect pulp capping in primary teeth. Am J Dent 2006;19:382-6.

27. Maltz M, Jardim JJ, Mestrinho HD, Yamaguti PM, Podestá K, Moura MS, et al. Partial removal of carious dentine: a multicenter randomized controlled trial and 18-month follow-up results. Caries Res 2013;47(2):103-9.

28. Maltz M, Moura M, Jardim JJ, Yamaguti PM, Araújo C, Paula L, et al. Partial removal of carious dentine: a multicenter, randomized, controlled trial. J. Dent. Res., Alexandria, v. 89, special issue B, 2010. Resumo do trabalho apresentado na 88th General Session \& Exhibition of IADR.

29. Maltz M, Garcia R, Jardim JJ, de Paula LM, Yamaguti PM, Moura MS, et al. Randomized Trial of Partial vs. Stepwise Caries Removal: 3-year Follow-up. J Dent Res. 2012;91(11):1026-31.

30. Koppe BTF. Remoção parcial de tecido cariado em dentes permanentes: acompanhamento de cinco anos [Trabalho de Conclusão de Curso]. Porto Alegre: Faculdade de Odontologia, Universidade Federal do Rio Grande do Sul; 2012.

31. Parolo CCF, Heller D, Bitello LF, Podestá K, Souza DCC, Hashizume L, et al. Effectiveness of the stepwise excavation treatment performed by dental students in Porto Alegre, Brazil. Caries Res 2007;41:268-334.
32. Busnello RG, Melchior R, Faccin C, Vettori D, Petter J, Moreira LB, et al. Characteristics associated with the dropout of hypertensive patients followed up in an outpatient referral clinic. Arq Bras Cardiol 2001;76(5):349-54.

33. Zanata RL, Navarro MF, Barbosa SH, Lauris JR, Franco EB. Clinical evaluation of three restorative materials applied in a minimal intervention caries treatment approach. J Public Health Dent 2003;63:221-6.

34. Weber C, Alves L, Maltz M. Treatment decisions for deep carious lesions in the Public Health Service in Southern Brazil. J Public Health Dent 2011;71(4):265-70.

35. Brasil. Ministério da Saúde. SB Brasil 2003: condições de saúde bucal da população brasileira 2002-2003. Resultados principais. Brasília; 2004 [acesso em jun. 2014]. Disponível em: <http://dab.saude.gov.br/cnsb/vigilancia.php $>$.

\section{Endereço para correspondência:}

Juliana Jobim Jardim

Rua Ramiro Barcelos, 2492

90035-003 Porto Alegre, RS

Telefone: (51) 3308-5193

Email: jujobim@yahoo.com

Recebido: 10/11/2014. Aceito: 13/04/2015. 